\title{
ON HOMEOMORPHISMS PRESERVING PRINCIPAL DIVISORS
}

\author{
YOSEF STEIN
}

\begin{abstract}
Let $S_{1}$ and $S_{2}$ be compact Riemann surfaces of genus $g>1$. Let $\tau$ : $S_{1} \rightarrow S_{2}$ be a continuous map. The map $\tau$ induces a group homomorphism from the group of divisors on $S_{1}$ into the group of divisors on $S_{2}$. This group homomorphism will be denoted by the same letter $\tau$ throughout this paper. If $D=\sum_{i=1}^{n} m_{i} p_{i}$ is a divisor on $S_{1}$, then $\tau(D)=\sum_{i=1}^{n} m_{i} \tau\left(p_{i}\right)$. If $\tau$ is a holomorphic or an anti-holomorphic homeomorphism, then $\tau(D)$ is a principal divisor on $S_{2}$ if $D$ is a principal divisor on $S_{1}$. To what extent is the converse of this statement true?

The answer to this question is provided by Theorem 1 of this paper: If $\tau(D)$ is a principal divisor on $S_{2}$ whenever $D$ is a principal divisor on $S_{1}$, then $\tau$ is either a holomorphic or an anti-holomorphic homeomorphism.
\end{abstract}

Let $\tau: S_{1} \rightarrow S_{2}$ be a surjective continuous map between compact Riemann surfaces of genus $g \geqslant 1$. Let $J\left(S_{1}\right)$ and $J\left(S_{2}\right)$ denote the corresponding Jacobi varieties and let $j_{1}: S_{1} \rightarrow J\left(S_{1}\right)$ and $j_{2}: S_{2} \rightarrow J\left(S_{2}\right)$ be Jacobi maps with basepoints $p_{0} \in S_{1}$ and $\tau\left(p_{0}\right) \in S_{2}$.

LEMMA 1. (1) The following statements are equivalent:

(i) $\tau(D)$ is a principal divisor on $S_{2}$ if $D$ is a principal divisor on $S_{1}$.

(ii) There is a group homomorphism $A: J\left(S_{1}\right) \rightarrow J\left(S_{2}\right)$ such that $A \circ j_{1}=j_{2} \circ \tau$.

(2) $A$ group homomorphism $A$ that satisfies (ii) is necessarily continuous, surjective and unique.

Proof. (1) Assume that $\tau$ satisfies (i). A mapping $A$ can be defined on the image $j_{1}\left(S_{1}\right) \subset J\left(S_{1}\right)$ as $j_{2} \circ \tau \circ j_{1}^{-1}$. This mapping can be extended upon the group $J\left(S_{1}\right)$ by additivity since points of $j_{1}\left(S_{1}\right)$ generate $J\left(S_{1}\right)$ as a group. Now we have to prove that $A$ is well defined. Let $\sum_{i=1}^{n} j_{1}\left(p_{i}\right)=\sum_{i=1}^{m} j_{1}\left(q_{i}\right)$ for $p_{i}, q_{i} \in S_{1}$. Then $p_{1}$ $+\cdots+p_{n}-q_{1}-\cdots-q_{m}+(m-n) p_{0}$ is a principal divisor on $S_{1}$. By our assumption this implies that $\tau\left(p_{1}\right)+\cdots+\tau\left(p_{n}\right)-\tau\left(q_{1}\right)-\cdots-\tau\left(q_{m}\right)+$ $(m-n) \tau\left(p_{0}\right)$ is a principal divisor on $S_{2}$. Therefore

$$
\begin{aligned}
A\left[\sum_{i=1}^{n} j_{1}\left(p_{i}\right)\right] & =\sum_{i=1}^{n} A j_{1}\left(p_{i}\right)=\sum_{i=1}^{n} j_{2} \tau\left(p_{i}\right) \\
& =\sum_{i=1}^{m} j_{2} \tau\left(q_{i}\right)=\sum_{i=1}^{m} A j_{1}\left(q_{i}\right)=A\left[\sum_{i=1}^{m} j_{1}\left(q_{i}\right)\right] .
\end{aligned}
$$

Thus $A: J\left(S_{1}\right) \rightarrow J\left(S_{2}\right)$ is a well-defined group homomorphism.

Now assume that there exists a group homomorphism $A: J\left(S_{1}\right) \rightarrow J\left(S_{2}\right)$ satisfying (ii). Let $D=p_{1}+\cdots+p_{n}-q_{1}-\cdots-q_{n}$ be a principal divisor on $S_{1}$.

Received by the editors August 25, 1980 and, in revised form, February 2, 1981.

1980 Mathematics Subject Classification. Primary 30F20; Secondary 32G15, 32G20. 
Then $\sum_{i=1}^{n} j_{1}\left(p_{i}\right)-\sum_{i=1}^{n} j_{1}\left(q_{i}\right)=0$. Applying $A$ we obtain the following:

$$
\begin{aligned}
0 & =A\left[\sum_{i=1}^{n} j_{1}\left(p_{i}\right)-\sum_{i=1}^{n} j_{1}\left(q_{i}\right)\right]=\sum_{i=1}^{n} A j_{1}\left(p_{i}\right)-\sum_{i=1}^{n} A j_{1}\left(q_{i}\right) \\
& =\sum_{i=1}^{n} j_{2} \tau\left(p_{i}\right)-\sum_{i=1}^{n} j_{2} \tau\left(q_{i}\right) .
\end{aligned}
$$

This implies that $\tau\left(p_{1}\right)+\cdots+\tau\left(p_{n}\right)-\tau\left(q_{1}\right)-\cdots-\tau\left(q_{n}\right)$ is a principal divisor on $S_{2}$.

(2) Let $A$ satisfy (ii). Then the following is a commutative diagram:

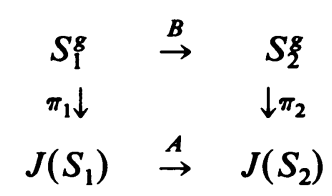

Here

$$
S_{k}^{g}=\overbrace{S_{k} \times S_{k} \times \cdots \times S_{k}}^{g} \quad(k=1,2) .
$$

$B\left(p_{1}, \ldots, p_{g}\right)=\left(\tau\left(p_{1}\right), \ldots, \tau\left(p_{g}\right)\right) ; \pi_{1}$ and $\pi_{2}$ are the natural maps induced by the Jacobi maps. Assume that $A$ is discontinuous at $x_{0} \in J\left(S_{1}\right)$. Then there exist an open set $V \subset J\left(S_{2}\right), V \ni A\left(x_{0}\right)$, and a sequence $\left\{x_{n}\right\}$ of points in $J\left(S_{1}\right)$ such that $\lim _{n \rightarrow \infty} x_{n}=x_{0}$ and $A\left(x_{n}\right) \notin V$ for all $n$. Since $\pi_{1}$ is surjective (by Jacobi Inversion Theorem) we can choose a sequence $\left\{t_{n}\right\}$ of points in $S_{1}^{8}$ such that $\pi_{1}\left(t_{n}\right)=x_{n}$. Since $S_{1}^{g}$ is compact, we can choose a converging subsequence $\left\{t_{n_{1}}\right\}$. Let $t_{0}=$ $\lim _{i \rightarrow \infty} t_{n_{i}}$. Then $\pi_{1}\left(t_{0}\right)=x_{0}$ since $\pi_{1}$ is continuous. Thus $\pi_{2} \circ B\left(t_{0}\right)=A \circ \pi_{1}\left(t_{0}\right)=$ $A\left(x_{0}\right)$. On the other hand

$$
\lim _{i \rightarrow \infty} A\left(x_{n_{i}}\right)=\lim _{i \rightarrow \infty} A \circ \pi_{1}\left(t_{n_{i}}\right)=\lim _{i \rightarrow \infty} \pi_{2} \circ B\left(t_{n_{i}}\right)=\pi_{2} \circ B\left(t_{0}\right)=A\left(x_{0}\right)
$$

since $\pi_{2} \circ B$ is a continuous map. This contradicts our earlier assumption that $V$ does not contain elements of the sequence $A\left(x_{n_{i}}\right)$. Hence $A$ is a continuous group homomorphism. Moreover, $A$ maps $j_{1}\left(S_{1}\right)$ onto $j_{2}\left(S_{2}\right)$ since $j_{1}$ and $j_{2}$ are homeomorphisms and $\tau$ is a surjective map. Therefore $A$ maps $J\left(S_{1}\right)$ onto $J\left(S_{2}\right)$ since points of $j_{2}\left(S_{2}\right)$ generate $J\left(S_{2}\right)$ as a group. The same argument (applied to $j_{1}$ instead of $j_{2}$ ) proves the uniqueness of $A$.

COROllary 1. If $\tau$ satisfies (i), then $\tau$ is real-analytic.

Proof. If $\tau$ satisfies (i), then by Lemma 1 there exists a continuous group homomorphism $A: J\left(S_{1}\right) \rightarrow J\left(S_{2}\right)$ such that $A \circ j_{1}=j_{2} \circ \tau$. This $A$ can be lifted to a real linear map $\tilde{A}: \mathbf{R}^{2 g} \rightarrow \mathbf{R}^{2 g}$ which makes the following diagram commutative:

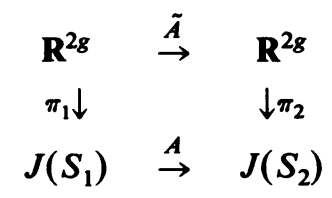

This is possible since $\mathbf{R}^{2 g}$ is the universal covering space for both $J\left(S_{1}\right)$ and $J\left(S_{2}\right)$, and since the only continuous group homomorphisms of $\mathbf{R}^{2 g}$ are linear maps. 
Therefore $A$ is real-analytic; and $\tau$ is real-analytic as well since $\tau=j_{2}^{-1} \circ A \circ j_{1}$ where both $j_{1}$ and $j_{2}$ are complex analytic homeomorphisms.

Corollary 2. Let $S_{1}$ and $S_{2}$ be compact Riemann surfaces of genus $g=1$. Then there exists a homeomorphism $\tau: S_{1} \rightarrow S_{2}$ such that $\tau$ and $\tau^{-1}$ satisfy (i).

Proof. $J\left(S_{1}\right)$ and $J\left(S_{2}\right)$ are one-dimensional complex analytic tori. Let $A$ be any continuous group isomorphism between them. Since $j_{1}\left(S_{1}\right)=J\left(S_{1}\right)$ and $j_{2}\left(S_{2}\right)=$ $J\left(S_{2}\right)$, we can define $\tau: S_{1} \rightarrow S_{2}$ to be $j_{2}^{-1} \circ A \circ j_{1}$. Then the pair $(A, \tau)$ will satisfy (ii) and, therefore, $\tau$ will satisfy (i). The same argument applies to $\tau^{-1}$.

This corollary implies that for any pair of Riemann surfaces of genus $g=1$, we can find a homeomorphism between them which preserves principal divisors. For $g \geqslant 2$ the situation is quite different. Let $\tau: S_{1} \rightarrow S_{2}$ be a surjective continuous map between compact Riemann surfces of genus $g \geqslant 2$. The group homomorphism $A$ that was introduced above can be lifted to a real $2 g \times 2 g$ matrix $\tilde{A}$ as described in the proof of Corollary 1 . We will identify $\mathbf{R}^{2 g}$ with $\mathbf{C}^{g}$ in the following way: Let $e_{1}, \ldots, e_{g}$ be the standard basis for $\mathbf{C}^{g}$, then the standard basis for $\mathbf{R}^{2 g}$ will be $e_{1}, i e_{1}, \ldots, e_{g}, i e_{g}$. The matrix $\tilde{A}$ can be considered thus as

$$
\left(\begin{array}{ccc}
A_{11} & \cdots & A_{1 g} \\
\vdots & & \vdots \\
A_{g 1} & \cdots & A_{g g}
\end{array}\right)
$$

where $A_{i j}$ 's are real $2 \times 2$ matrices. Let $p \in S_{1}$ and let $z$ denote a local analytic coordinate in a neighbourhood of $p$; let $z=x+i y$. Let $w=u+i v$ be a local analytic coordinate in a neighbourhood of $\tau(p)$. The bases for holomorphic differentials $\left\{\omega_{k}\right\}$ and $\left\{\tilde{\omega}_{k}\right\}$ will be considered as column vectors

$$
\omega=\left(\begin{array}{c}
\omega_{1} \\
\vdots \\
\omega_{g}
\end{array}\right) \text { and } \tilde{\omega}=\left(\begin{array}{c}
\tilde{\omega}_{1} \\
\vdots \\
\tilde{\omega}_{g}
\end{array}\right)
$$

( $\omega$ corresponds to $S_{1}$ and $\tilde{\omega}$ corresponds to $S_{2}$ ). With respect to the local coordinates $z$ and $w$,

$$
\begin{aligned}
& \omega_{k}=\left(f_{k}+i h_{k}\right) d z=\left(f_{k} d x-h_{k} d y\right)+i\left(h_{k} d x+f_{k} d y\right), \\
& \tilde{\omega}_{k}=\left(\tilde{f}_{k}+i \tilde{h}_{k}\right) d w=\left(\tilde{f}_{k} d u-\tilde{h}_{k} d v\right)+i\left(\tilde{h}_{k} d u+\tilde{f}_{k} d v\right) .
\end{aligned}
$$

Here $f_{k}, h_{k}, \tilde{f}_{k}$ and $\tilde{h}_{k}$ are real-valued local functions. $\omega_{k}$ and $\tilde{\omega}_{k}$ can be considered thus as pairs of real-valued differential forms on the underlying differentiable structures of $S_{1}$ and $S_{2}$. Let $L_{k}$ denote a matrix

and let $\tilde{L_{k}}$ denote a matrix

$$
\left(\begin{array}{cc}
f_{k} & -h_{k} \\
h_{k} & f_{k}
\end{array}\right)
$$

$$
\left[\begin{array}{cc}
\tilde{f}_{k} & -\tilde{h}_{k} \\
\tilde{h}_{k} & \tilde{f}_{k}
\end{array}\right]
$$


Throughout this paper we will consider $\mathbf{C}$ as a subalgebra of the algebra of all real $2 \times 2$ matrices, and we will identify a complex number $a+i b$ with the real matrix

$$
\left(\begin{array}{cc}
a & -b \\
b & a
\end{array}\right)
$$

Complex-valued functions will be considered as real matrix-valued functions. By Corollary 1 to Lemma $1 \tau$ is real-analytic. Let

$$
K=\left(\begin{array}{ll}
\frac{\partial u}{\partial x} & \frac{\partial u}{\partial y} \\
\frac{\partial v}{\partial x} & \frac{\partial v}{\partial y}
\end{array}\right)
$$

be the local Jacobian matrix corresponding to $\tau$ and $x, y, u$ and $v$.

LEMMA 2. Let $\tau: S_{1} \rightarrow S_{2}, A$, $\omega$ and $\tilde{\omega}$ be as above. Then

(1) for each $k\left(\tilde{L}_{k} \circ \tau\right) K=\sum_{s=1}^{g} A_{k s} L_{s}$.

(2) $A$ and $\tau$ are local homeomorphisms.

Proof. (1) The Jacobi mappings $j_{1}$ and $j_{2}$ can be considered as real-analytic maps from the two-dimensional real surfaces $S_{1}$ and $S_{2}$ into $J\left(S_{1}\right)$ and $J\left(S_{2}\right)$ respectively. The Jacobian matrix of $j_{1}$ in terms of the local coordinates $(x, y)$ is

$$
\left(\begin{array}{c}
L_{1} \\
\vdots \\
L_{8}
\end{array}\right)
$$

Similarly, the Jacobian matrix of $j_{2}$ in terms of the local coordinates $(u, v)$ is

$$
\left(\begin{array}{c}
\tilde{L}_{1} \\
\vdots \\
\tilde{L}_{g}
\end{array}\right)
$$

The Jacobian matrix of $A$ is $\tilde{A}$ and since $A \circ j_{1}=j_{2} \circ \tau$, we obtain

$$
\left[\begin{array}{c}
\left(\tilde{L}_{1} \circ \tau\right) K \\
\vdots \\
\left(\tilde{L}_{g} \circ \tau\right) K
\end{array}\right)=\tilde{A}\left(\begin{array}{c}
L_{1} \\
\vdots \\
L_{g}
\end{array}\right) .
$$

(2) $A$ is surjective by Lemma 1 . Therefore $\tilde{A}$ is surjective and hence of rank $2 g$. Since

$$
\mathrm{rk}\left(\begin{array}{c}
L_{1} \\
\vdots \\
L_{8}
\end{array}\right)=2
$$


at every point of $S_{1}$ and

$$
\mathrm{rk}\left(\begin{array}{c}
\tilde{L}_{1} \\
\vdots \\
\tilde{L}_{g}
\end{array}\right]=2
$$

at every point of $S_{2}$, equation (1) implies that det $K$ is never 0 . Now we can prove the main theorem.

THEOREM 1. Let $\tau: S_{1} \rightarrow S_{2}$ be a surjective continuous map between compact Riemann surfaces of genus $g \geqslant 2$. If $\tau(D)$ is a principal divisor on $S_{2}$ whenever $D$ is a principal divisor on $S_{1}$, then

(1) $\tau$ is either holomorphic or anti-holomorphic.

(2) $\tau$ is a homeomorphism.

Proof. By assertion (1) of Lemma 2 we have $g$ equations of the form $\left(\tilde{L}_{k} \circ \tau\right) K$ $=\sum_{s=1}^{g} A_{k s} L_{s}$. These equations depend on our choice of the local coordinates $x, y$, $u$ and $v$. To get rid of this we will multiply (from the right) $\left(\tilde{L}_{k} \circ \tau\right) K$ by $K^{-1}\left(\tilde{L}_{1} \circ \tau\right)^{-1}$ and $\sum_{s=1}^{g} A_{k s} L_{s}$ by $\left(\sum_{s=1}^{g} A_{1 s} L_{s}\right)^{-1}$. Then we obtain $g-1$ equations of the form

$$
\left(\tilde{L}_{k} \tilde{L}_{1}^{-1}\right) \circ \tau=\left(\sum_{s=1}^{g} A_{k s} L_{s}\right)\left(\sum_{s=1}^{g} A_{1 s} L_{s}\right)^{-1}
$$

Perhaps some explanations are necessary at this point. First, $\tilde{L}_{1} \circ \tau$ is a local map from $S_{1}$ into the algebra of real $2 \times 2$ matrices. $\left(\tilde{L}_{1} \circ \tau\right)^{-1}$ means taking the inverse of a value of this matrix-valued function: $\left(\tilde{L}_{1} \circ \tau\right)^{-1}=\left(\tilde{L}_{1}^{-1}\right) \circ \tau$. Second, the matrix $\tilde{L}_{1}$ is either invertible or 0 ; and we take the inverse at all points except those for which $\tilde{L}_{1}$ is 0 (such points form the divisor of $\tilde{\omega}_{1}$ ). Third, by assertion (2) of Lemma 2 det $K$ is never 0 and $K^{-1}$ always exists.

Returning to our proof, let us consider any one of the equations obtained above. The left part of it has the form $\left(\tilde{L}_{k} \tilde{L}_{1}^{-1}\right) \circ \tau$. But $\tilde{L}_{k} \tilde{L}_{1}^{-1}=\tilde{\omega}_{k} / \tilde{\omega}_{1}$ which is a global meromorphic function on $S_{2}$. Let us denote this function by $\tilde{F}_{k} \cdot\left(\tilde{L}_{k} \tilde{L}_{1}^{-1}\right) \circ \tau$ is, therefore, the pull-back of this function by $\tau$. The right part of our equation is a matrix of the form $\left(\sum_{s=1}^{g} A_{k s} L_{s}\right)\left(\sum_{s=1}^{g} A_{1 s} L_{s}\right)^{-1}$. But this matrix can be written as $\left(\sum_{s=1}^{g} A_{k s} L_{s} L_{1}^{-1}\right)\left(\sum_{s=1}^{g} A_{1 s} L_{s} L_{1}^{-1}\right)^{-1} . L_{s} L_{1}^{-1}$ is a global meromorphic function on $S_{1}$. Let us denote this function by $F_{s}$. Thus we have $g-1$ equations of the form

$$
\tilde{F}_{k} \circ \tau=\left(\sum_{s=1}^{g} A_{k s} F_{s}\right)\left(\sum_{s=1}^{g} A_{1 s} F_{s}\right)^{-1} \text { or }\left(\tilde{F}_{k} \circ \tau\right) \sum_{s=1}^{g} A_{1 s} F_{s}=\sum_{s=1}^{g} A_{k s} F_{s}
$$

It should be mentioned one more time that we consider complex numbers as a subalgebra of the algebra of real $2 \times 2$ matrices. These equations are considered in the point-wise sense; and they are valid for all points of $S_{1}$ that are not poles for any of $F_{k}$ 's and such that their images in $S_{2}$ are not poles for any of $\tilde{F}_{k}$ 's. Since $\tau$ is a local homeomorphism, there is only a finite set of such exceptional points. 
Let us consider now a complex-valued real-linear functional $T$ that can be defined on the algebra of real $2 \times 2$ matrices as follows:

$$
T\left(\begin{array}{ll}
a & b \\
c & d
\end{array}\right)=a-d+i(b+c) .
$$

This functional has the following property:

$$
T\left[\left(\begin{array}{cc}
\alpha & -\beta \\
\beta & \alpha
\end{array}\right)\left(\begin{array}{ll}
a & b \\
c & d
\end{array}\right)\right]=(\alpha+i \beta) T\left(\begin{array}{ll}
a & b \\
c & d
\end{array}\right)
$$

and

$$
T\left[\left(\begin{array}{ll}
a & b \\
c & d
\end{array}\right)\left(\begin{array}{cc}
\alpha & -\beta \\
\beta & \alpha
\end{array}\right)\right]=(\alpha-i \beta) T\left(\begin{array}{ll}
a & b \\
c & d
\end{array}\right) .
$$

Furthermore, the kernel of $T$ consists of those matrices which correspond to complex numbers. Let us apply $T$ to the equation (2). We obtain the following:

$$
\left(\tilde{F}_{k} \circ \tau\right) \sum_{s=1}^{g} T\left(A_{1 s}\right) \bar{F}_{s}=\sum_{s=1}^{g} T\left(A_{k s}\right) \bar{F}_{s} \text {. }
$$

Suppose that $T\left(A_{k s}\right)=0$ for all indices $k$ and $s$. Then all matrices $A_{k s}$ are merely complex numbers $c_{k s}$ and the equation (2) implies that

$$
\tilde{F}_{k} \circ \tau=\frac{\sum_{s=1}^{g} c_{k s} F_{s}}{\sum_{s=1}^{g} c_{1 s} F_{s}}
$$

This means that $\tilde{F}_{k} \circ \tau$ is a meromorphic function on $S_{1}$ and, therefore, $\tau$ is holomorphic. If not all of $T\left(A_{k s}\right)$ are equal to 0 , then the equation (3) implies that

$$
\tilde{F}_{k} \circ \tau=\frac{\sum_{s=1}^{g} T\left(A_{k s}\right) \bar{F}_{s}}{\sum_{s=1}^{g} T\left(A_{1 s}\right) \bar{F}_{s}}
$$

( $T\left(A_{k s}\right)$ is a complex number). This means that $\tau$ is anti-holomorphic. Finally, since $\tau$ is either a holomorphic or an anti-holomorphic local homeomorphism it must be a global homeomorphism (since it has no branch points and $g>1$ ).

COROllary. Let $S_{1}$ and $S_{2}$ be compact Riemann surfaces of genus $g>2$ with Jacobi mappings $j_{1}$ and $j_{2}$. Let $A: J\left(S_{1}\right) \rightarrow J\left(S_{2}\right)$ be a continuous group homomorphism such that $A j\left(S_{1}\right)=j_{2}\left(S_{2}\right)$. Then

(1) $A$ is either holomorphic or anti-holomorphic.

(2) $A$ is a group isomorphism.

The proof is obvious.

\section{BIBLIOGRAPHY}

1. R. Gunning, Lectures on Riemann surfaces, Princeton Univ. Press, Princeton, N.J., 1967.

2. __ Lectures on Riemann surfaces, Jacobi varieties, Princeton Univ. Press, Princeton, N. J., 1972.

Department of Mathematics, University of California, Santa Cruz, California 95060

Current address: 25/13 Ha Thia Street, Rehovot, Israel 